\title{
Gobernabilidad y atención comunitaria en situación de emergencia en un distrito de Lima
}

\author{
Ruben Ángel Rivera Uceda \\ rriverau@ucvvirtual.edu.pe \\ https://orcid.org/0000-0001-9676-4686 \\ Universidad Cesar Vallejo \\ Lima - Perú
}

\section{RESUMEN}

La gobernabilidad en América Latina ha sido otra víctima de la pandemia, que no sólo ha desencadenado una profunda crisis económica con un notable incremento de la pobreza y la desigualdad, sino también ha situado la recuperación de la estabilidad políticoinstitucional como uno de los principales desafíos que debe afrontar la región a corto plazo.

La investigación es cuantitativo, no experimental de naturaleza transversal, básica. Conformado por 108 funcionarios de la gestión municipal. El objetivo general Determinar la relación que existe entre gestión municipal y la gobernabilidad en la atención comunitaria en una municipalidad distrital de Lima.

Llegando a la conclusión: Al hacer el contraste de la hipótesis general usando técnica estadística predictiva del SPSS V23, existen evidencias suficientes para afirmar que la gobernabilidad tiene relación positiva y significativa con la gestión municipal ( $\mathrm{rs}=0.823$, p< 0.05). Una Administración fuerte no debería ser una Administración sobredimensionada, intrusiva o gigantesca, sino firme en la defensa del bien común, independiente frente a grupos de interés espurios y dinámica para impulsar el desarrollo de sus territorios. Todo ello es posible y necesario para afrontar los retos del nuevo siglo; y todo ello es compatible con dejar atrás modelos obsoletos que ya no tienen sentido.

Palabras clave: gobernación; atención comunitaria; política; capacidad. 


\title{
Governance and community care in an emergency situation in a district of Lima
}

\begin{abstract}
Governance in Latin America has been another victim of the pandemic, which has not only triggered a deep economic crisis with a notable increase in poverty and inequality but has also placed the recovery of political-institutional stability as one of the main challenges to be faced by the region in the short term.

The research is quantitative, non-experimental, cross-sectional, basic in nature. Conformed by 108 municipal management officials. The general objective proposed is to determine the relationship that exists between city management that is not significantly related to governance.

Reaching the conclusion: When contrasting the general hypothesis using SPSS V23 predictive statistical technique, there is sufficient evidence to affirm that governance has a positive and significant relationship with municipal management $(r s=0.823, p<0.05)$. A strong administration should not be an oversized, intrusive, or gigantic administration, but one that is firm in the defense of the common good, independent in the face of spurious interest groups and dynamic in promoting the development of its territories. All this is possible and necessary to face the challenges of the new century; and all this is compatible with leaving behind obsolete models that no longer make sense.
\end{abstract}

Keywords: governance; community care; policy; capacity.

Artículo recibido: 30 noviembre. 2021 Aceptado para publicación: 29 diciembre 2021 Correspondencia: rriverau@ucvvirtual.edu.pe Conflictos de Interés: Ninguna que declarar 


\section{INTRODUCCIÓN}

El presente proyecto de investigación denominado Gobernabilidad y atención comunitaria en situación de emergencia en un distrito de Lima, nace como una necesidad de proporcionar información que se relacione con el desarrollo de órganos y permitan que se brinde conocimientos, ya sea a la ciudadanía como a las diversas empresas públicas y privadas que se interesen por entender los procesos que se desarrollan y las decisiones que se toman a nivel de los concejales, o en un plenario de la ciudad, así como los documentos de respaldo que permitan tomar estas decisiones. Según Alcántara (2014), "Es concebible que una situación en la que exista un conjunto de condiciones favorables para el funcionamiento del gobierno se inscriba en su entorno o naturaleza" (p. 39). Arbos (1993) citado por Alcántara (1995), Se menciona que la gobernabilidad es "la característica de una sociedad política, donde sus instituciones de gobierno operan efectivamente en su espacio de una manera que los ciudadanos ven como legítima, permitiendo el ejercicio de la libertad política del poder ejecutivo a través de la obediencia a los ciudadanos del municipio". (p. 40). Citando a Camou (2001), planteó que es importante que La gobernabilidad se entiende como "el estado de equilibrio dinámico entre el nivel de necesidades sociales y la capacidad del sistema político (estado / gobierno) para satisfacerlas de manera legal y efectiva" (Mayorga y Córdoba, 2007, p.1). En otras palabras, la gobernabilidad puede ser una característica de una sociedad política, en la que el ejercicio del poder involucra una serie de mecanismos y procesos que benefician a todos los intereses de la sociedad. Prats (2001) señala que las crisis de gobernabilidad pueden surgir debido a la incapacidad de las reglas y procedimientos para abordar los problemas de interacción (acción colectiva) de los actores poderosos, especialmente cuando el equilibrio es alto. Barroso (2016), "La gobernabilidad vista desde el liderazgo socio organizativo de la asociación de mujeres Waorani de la amazonia ecuatoriana Amwae (2010-2012)”. Citando a Gutierrez y Giraldo (2016), la justicia social a través de la gobernanza global es de suma importancia para el ser humano y el respeto a su dignidad y el pleno reconocimiento de sus derechos a través de capacidades empoderadoras que son la fuente de las necesidades de una sociedad justa. Según Storner (1996) La organización es la segunda etapa de la Gobernanza para actuar en que se ordenan aspectos del Consejo de Administración, básicamente, básicamente, los costos 
de inversión, el tiempo de desarrollo de la operación, así como los trabajadores especializados necesarios para garantizar que los resultados puedan ser positivos.

En ese sentido, mediante la recolección de datos que se tomará con una encuesta con preguntas por variable, la población consta de los trabajadores de la municipalidad, la muestra son los funcionarios de la gerencia de la institución en estudio, la conclusión es saber la capacidad de organización desde donde se puede definir que es uno de los pilares de la administración pública, puesto que se busca poner en orden las cosas para llegar al objetivo, en este caso la gestión municipal no escapa a la organización de los procesos administrativos para la cual los responsables designados de cada área facilitan el establecimiento de metas. En relación con la realidad problemática que se vive en un mundo globalizado, que genera un veloz desarrollo en tecnologías de información, competencia desenfrenada, necesidad de reducir costes y estrés hacia el cliente, calidad total y necesidad de competitividad que se constituyen en no se pueden ignorar las poderosas influencias. Entonces, si el mundo cambia, también lo hacen las empresas, también deberían hacerlo los recursos humanos, de manera paralela acompañándolos. Cada vez, más organizaciones ponen al frente al área de Recursos Humanos direccionando un cambio organizacional; en otras, impide el ajuste a nuevas condiciones de modernidad, convirtiéndose en un obstáculo para la organización.

En América Latina, una experiencia analizada mostró que las transferencias de formas de gobierno de parte de los organismos internacionales no produjeron los resultados que se esperaban en términos de alcanzar políticas civiles democráticas efectivas y más efectivas. La mayor debilidad significa cambiar las recomendaciones de una ciudad, región o país a otro, de manera dogmática. Ignorar los procesos históricos y / o las relaciones. Las relaciones de poder compartidas apoyan la toma de decisiones vinculante. que se aplican en relación con la sociedad. Un municipio, por su cercanía al ciudadano, debe mantener el trabajo eficaz y eficaz confiere legitimidad directa a las personas. La democracia moderna no solo implicará la participación en las elecciones mediante el voto, sino que sobre todo implicará la participación en el proceso de votación que se realizan para deliberar, para implementar y para evaluar la gestión y las políticas locales. Todo ello requiere de diferentes e innovadoras formas de gestión municipal. Los ciudadanos se encuentran hartos de los municipios que generan más gastos y proporcionan cada vez menos servicios, debido a la frustración con la burocracia incontrolable. Actualmente la 
gestión municipal en el Perú se rige por las normas emitidas por el Estado, sin embargo no escapa a la realidad de los conceptos de la teoría sobre la administración en general, el análisis de la problemática no radica en la antigüedad de los procesos o en la modernidad de las aplicaciones y avances tecnológicos en materia administrativa; el problema radica en esta concepción humana, es decir la problemática recae en las personas que asumen el control de la administración por un periodo determinado y que son dirigidos en los mandos superiores por el denominado cargos de confianza. Actualmente la gestión municipal en el Perú, se rige por las normas emitidas por el Estado, sin embargo no escapa a la realidad de los conceptos de la teoría sobre la administración en general, el análisis de la problemática no radica en la antigüedad de los procesos o en la modernidad de las aplicaciones y avances tecnológicos en materia administrativa; el problema radica en esta concepción humana, es decir la problemática recae en las personas que asumen el control de la administración por un periodo determinado y que son dirigidos en los mandos superiores por el denominado cargos de confianza. En ese sentido, planteamos las siguientes preguntas que servirán como guía para la mejor comprensión y entendimiento de lo que se pretende abarcará en la investigación, a saber: En forma general: ¿De qué manera la gestión municipal se relaciona con la gobernabilidad en la atención comunitaria en una municipalidad distrital de Lima?: Teniendo como problemas específicos: ¿De qué manera la planeación de la gestión Municipal se relaciona con la gobernabilidad en la atención comunitaria en una municipalidad distrital de Lima?,¿De qué manera la organización de la Gestión Municipal con la gobernabilidad en la atención comunitaria en una municipalidad distrital de Lima?, ¿De qué manera la dirección de la gestión municipal se relaciona con la gobernabilidad en la atención comunitaria en una municipalidad distrital de Lima?.

Formulando el objetivo general: Determinar la relación que existe entre gestión municipal y la gobernabilidad en la atención comunitaria en una municipalidad distrital de Lima. Posteriormente el objetivo específico: Determinar la relación que existe entre la planeación de la gestión Municipal y la gobernabilidad en la atención comunitaria en una municipalidad distrital de Lima, Determinar la relación que existe entre la organización de la gestión Municipal y la gobernabilidad en la atención comunitaria en una municipalidad distrital de Lima, Determinar la relación que existe entre la dirección de la 
gestión Municipal y la gobernabilidad en la atención comunitaria en una municipalidad distrital de Lima.

El trabajo encuentra su justificación teórica En ese sentido la presente investigación se realiza tomando como base trabajos similares a nivel nacional e internacional, que permiten analizar las variables aplicadas a una municipalidad distrital, los resultados determinarán la relación actual que tiene la gobernabilidad en la atención comunitaria en una municipalidad distrital, permitiendo que se mejore la gestión a nivel directivo en la consideración a las competencias de las personas dirigido al logro de objetivos institucionales. Asimismo, la presente investigación es práctica porque permitir que la gestión a nivel de dirección reconozca la importancia que tiene la Gestión Municipal y la gobernabilidad en la atención comunitaria en una municipalidad distrital de Lima, en el logro de objetivos institucionales; permitiendo que elaboren estrategias y competencias de las personas que se sientan identificados con su institución. Partiendo de la formulación del problema para plantear la hipótesis: H1: La Gestión Municipal se relaciona significativamente con la gobernabilidad. Ho: La Gestión Municipal no se relaciona significativamente con la gobernabilidad.

\section{ESTRATEGIAS METODOLÓGICAS O MATERIALES Y MÉTODOS}

El estudio en antecedentes aplicable es básico, porque en fundamentación en artículos teóricos para determinar la relación entre variables. Asimismo, por su nivel de investigación es correlacional, porque en sus categorías de estudio que se aspira encontrar el nivel el referente entre las 2 variables mediante los datos recogidos.

Vargas (2009) la define como: "Se la conoce también como investigación básica, que trata el tema de la investigación sin considerar la aplicación inmediata, pero teniendo en cuenta los resultados, descubrimientos, nuevos productos y avances científicos que puedan aparecer" (p. 6).

Diseño de la investigación: Investigación no experimental de naturaleza transectorial (transversal). $1^{\circ}$ es no experimental, ya que las variables no se modifican, y de igual manera, el estudio se basará en la observación y análisis del estado del medio natural sin modificación, es decir se obtiene la información de la unidad de estudio tal como se da en la realidad, el investigador no interviene en la situación que se desarrolla. $2^{\circ}$, es transversal porque la encuesta que se almacena es en un momento dado, es decir, no se desarrolla un estudio ni antes y después. Hernández (2014), afirmó que "correlacionan 
variables en un patrón predecible a un grupo o población” (p. 93). Tamayo (2003), el procedimiento de operacionalización es fundamental identifica los criterios de evaluación a comenzar las cuales se determinará la relación entre las variables expuestas, es imprescindible tener en cuenta: determinación nominal (variable medir), definir operacionalmente la dimensión (factor a medir) parámetro (como medir), indicador (importancia, \%). Según Rodríguez y Gutierrez (2005) Afirma que "una población es el conjunto de medidas que se pueden realizar sobre una propiedad común de un grupo de organismos u organismos”. (pág.79). La población está contabilizada y estará conformada por la comunidad de emergencia de un distrito de Lima. Esta investigación, estuvo representada por 108 personas seleccionadas de manera aleatoria entre el personal de una municipalidad distrital de Lima. El resultado, la encuestas a 108 personas, el 0.95 de las veces del número que se quiere medir estará en el intervalo \pm 0.5 respecto a los datos que se observa en el cuestionario. Para nuestro estudio las muestras obtenidas fueron de 30 trabajadores de una municipalidad distrital de Lima la técnica estadística empleada para la obtención de la muestra fue la no probabilística, porque según Hernández, Fernández y Baptista (2014, p.176) se indica que la elección de los elementos no depende de la probabilidad, sino de causas relacionadas con las características de la investigación o los propósitos del investigador Usuarios de una Municipalidad Distrital de Lima Administrativos con conocimiento de herramientas informática. Usuarios que no pertenecen a la Municipalidad Provincial de Lima. Por técnica se entenderá según Arias (2006), "El procedimiento o forma particular de obtener datos o información” (p. 67) Es decir, indica cómo se recopilan los datos. En la encuesta, la encuesta se utilizará como método de recopilación de datos; Según Corral (2008), "La indagación y la observación son dos técnicas básicas para la recolección de datos primarios cuantitativos y cualitativos en la investigación científica. Las principales herramientas utilizadas por estas técnicas son: cuestionarios, entrevistas y fórmulas de observación". (p. 153). De la encuesta, donde se utilizaron dos cuestionarios con escalas tipo Likert para la muestra de trabajadores. Instrumentos de recolección de datos La fuente principal es la fuente principal de información recopilada por el investigador para el servicio de investigación. Al igual que con la encuesta actual, el Cuestionario de actitudes de la escala Likert se utilizará como herramienta de recopilación de datos.

Menéndez (2016) La validez del cuestionario es la calidad del instrumento que mide el 
rasgo o rasgo que se va a medir. Con la validación, se trata de determinar si el cuestionario mide realmente el propósito para el que fue creado (pág.25). Según Arias (2006), define que es la herramienta de recolección de datos se define como "cualquier recurso, dispositivo o formato (papel o digital), que se utiliza para recolectar, registrar o almacenar información". Así, esta herramienta resume el trabajo de investigación, por lo que los datos corresponden a indicadores de los tipos de información utilizados teniendo en cuenta las fuentes de información utilizadas para recopilar los datos del Proyecto, se basan en la consulta con fuentes primarias y secundarias y la observación directa. La fuente principal es la fuente principal de información recopilada por el investigador para el servicio de investigación. Al igual que con la encuesta actual, el Cuestionario de actitudes de la escala Likert se utilizará como herramienta de recopilación de datos. Es una herramienta que permite la recolección de información estructurada a través de una serie de preguntas predefinidas y codificadas. Respecto al cuestionario, Sierra (1994) especifica que “... esta herramienta consiste en aplicar una serie de preguntas o ítems sobre un determinado problema de investigación que queremos plantear saber algo a un mundo específico de individuos". En esta encuesta, se utilizaron cuestionarios como herramienta de recopilación de datos. Según Hernández, et al (2010), La confiabilidad de la herramienta "es el grado en que una herramienta produce resultados consistentes y consistentes". (pág. 200). Según Ander (2002), la confiabilidad ayuda a cuantificar que el instrumento utilizado mide lo que se requiere. Para determinar la confiabilidad del instrumento, primero consideramos que el instrumento tiene cinco respuestas, por lo que es poliatómico, por lo que el coeficiente alfa de Cronbach se determinó mediante el instrumento estadístico. De igual forma para determinar el nivel de confianza se utilizará la siguiente tabla de (Bolívar, 2002). El método utilizado en este estudio es el método de inferencia hipotética, y al respecto Bernal (2006) afirma que "este método consiste en un procedimiento que comienza con el número de hechos encontrados" (p. 56).

La prueba de hipótesis debe realizarse teniendo en cuenta los siguientes criterios: (a) formular una hipótesis nula o activa y una hipótesis alternativa o de investigación, (b) determinar el nivel de significancia o error que el investigador quiere asumir, (c) elegir el estadístico de prueba, (d) estimar el valor probabilístico, y (e) Tomar una decisión, dependiendo del resultado. En la presente investigación se utilizó como técnica de recojo de información, la encuesta, la cual se define como: "una técnica que se utiliza en la 
investigación de mercados, y consiste en recoger la información, de las personas que tienen similar característica, a través de preguntas que se redactan en base a los indicadores establecidos" (Hernández, et al., 2014). En la presente investigación el cuestionario de preguntas fue el instrumento que permitió recoger información directamente de las unidades de análisis. Utilizando como definición que: "Un cuestionario, es un documento, donde se redactan un grupo de preguntas, respecto a los indicadores que se quieren medir, y se vinculan a las variables de estudio a partir de las dimensiones de la investigación. Cada una de estas preguntas debe guardar relación con el planteamiento de los problemas y de las hipótesis. (Hernández et al.,2014). La validez y confiabilidad de instrumentos están referidos al grado con el cual, el "instrumento mide lo que pretende medir"; para asegurar ello, los instrumentos en esta investigación debían ser confiables y válidos, por ello se sometieron al proceso de validación de contenido. la prueba estadística de confiabilidad Alfa de Crombach un 0,95\% para la primera variable y $0.93 \%$ para la segunda variable; este resultado indicó la alta confiabilidad que permitió aplicar los instrumentos en la investigación.

La presente investigación dio inicio con la explicación y narrativa de la realidad problemática, con el apoyo de trabajos previos y/o antecedentes. Se desarrollaron las teorías vinculadas por cada una de las variables en estudio, con fuentes de información preferentemente que no excedan una antigüedad de los 5 años, a excepción de las teorías existentes. Seguidamente se determinó la metodología a seguir, la población y muestra a considerar, la operacionalización y elaboración de los instrumentos que se presentaron para la técnica de la encuesta, entre otros. Las encuestas se aplicaron personal de una municipalidad Distrital de Lima, los datos recopilados se procesaron para obtener los resultados sin ningún tipo de adulteración en el proceso, para obtener los resultados, las conclusiones y las recomendaciones. Previamente al recojo y organización de información se procedió a codificarlos datos en la matriz de tabulación de doble entrada empleando el Software especializado de estadística PSS versión 26.0. Seguidamente, aplicando la estadística descriptiva, se analizaron los resultados y se calculó la frecuencia relativa y simple. Estos resultados se presentaron en tablas bidimensionales y en gráficos de barras, utilizando el software Office Excel 2016.

Asimismo, el análisis descriptivo, se efectuó mediante tablas cruzadas de frecuencia, figuras e interpretaciones considerando los objetivos propuestos y el análisis inferencial 
se efectuó mediante una prueba de hipótesis, utilizando el coeficiente RHO de Spearman que permitió conocer la correlación entre las variables. Esta investigación ha respetado la confidencialidad de datos, así como las opiniones vertidas por los encuestados en los instrumentos, los cuales han sido procesados adecuadamente sin adulteraciones. Asimismo, por cuestiones éticas no se mencionaron los nombres de los funcionarios que han constituido las unidades de análisis de la investigación. Dicha información es de privacidad del investigador, además, fue necesario elaborar los documentos de consentimiento informado a cada persona encuestada, donde autorizan su conocimiento del trabajo investigativo como: los objetivos de estudio, el uso que se hará de los datos que proporcionen, la forma en la que se difundirán los resultados y las características necesarias para que ellos participen y tomen decisiones informadas al acceder o no a participar en el estudio y declaren por escrito de manera explícita su consentimiento de participar, donde no se recibió ninguna respuesta y se dio por aceptada la toma de encuestas a dichos funcionarios.

\section{RESULTADOS Y DISCUSIÓN}

\section{Resultados descriptivos}

Tabla 1 Niveles de percepción de Planificación

\begin{tabular}{clcc}
\hline & Planificación & Frecuencia & Porcentaje \\
\hline \multirow{4}{*}{ Válido } & Pésimo & 21 & 19,4 \\
& Deficiente & 21 & 19,4 \\
& Bueno & 38 & 35,2 \\
& Sobresaliente & 28 & 25,9 \\
& Total & 108 & 100,0 \\
\hline
\end{tabular}

Figura 1 Planificación

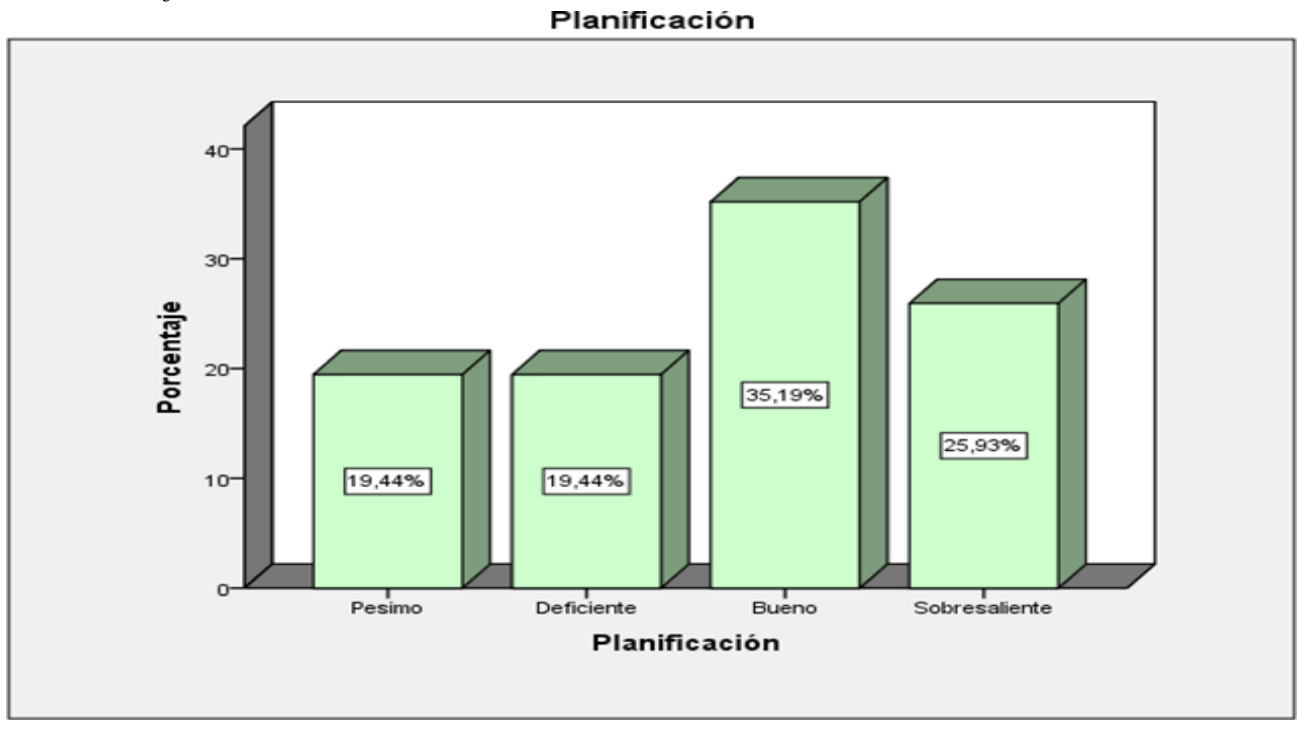


Según las Figuras 1 y 9 de los datos, el 19,4\% la calificó como mala en términos de planificación, el 19,4\% como mala, el 35,2\% como buena y el 25,9\% como excepcional, predominando el ajuste de la planificación.

\section{Tabla 2}

Niveles de percepción de Organización

\begin{tabular}{clcc}
\hline & Organización & Frecuencia & Porcentaje \\
\hline \multirow{4}{*}{ Válido } & Pésimo & 20 & 18,5 \\
& Deficiente & 28 & 25,9 \\
& Bueno & 36 & 33,3 \\
& Sobresaliente & 24 & 22,2 \\
& Total & 108 & 100,0 \\
\hline
\end{tabular}

\section{Figura 2}

\section{Organización}

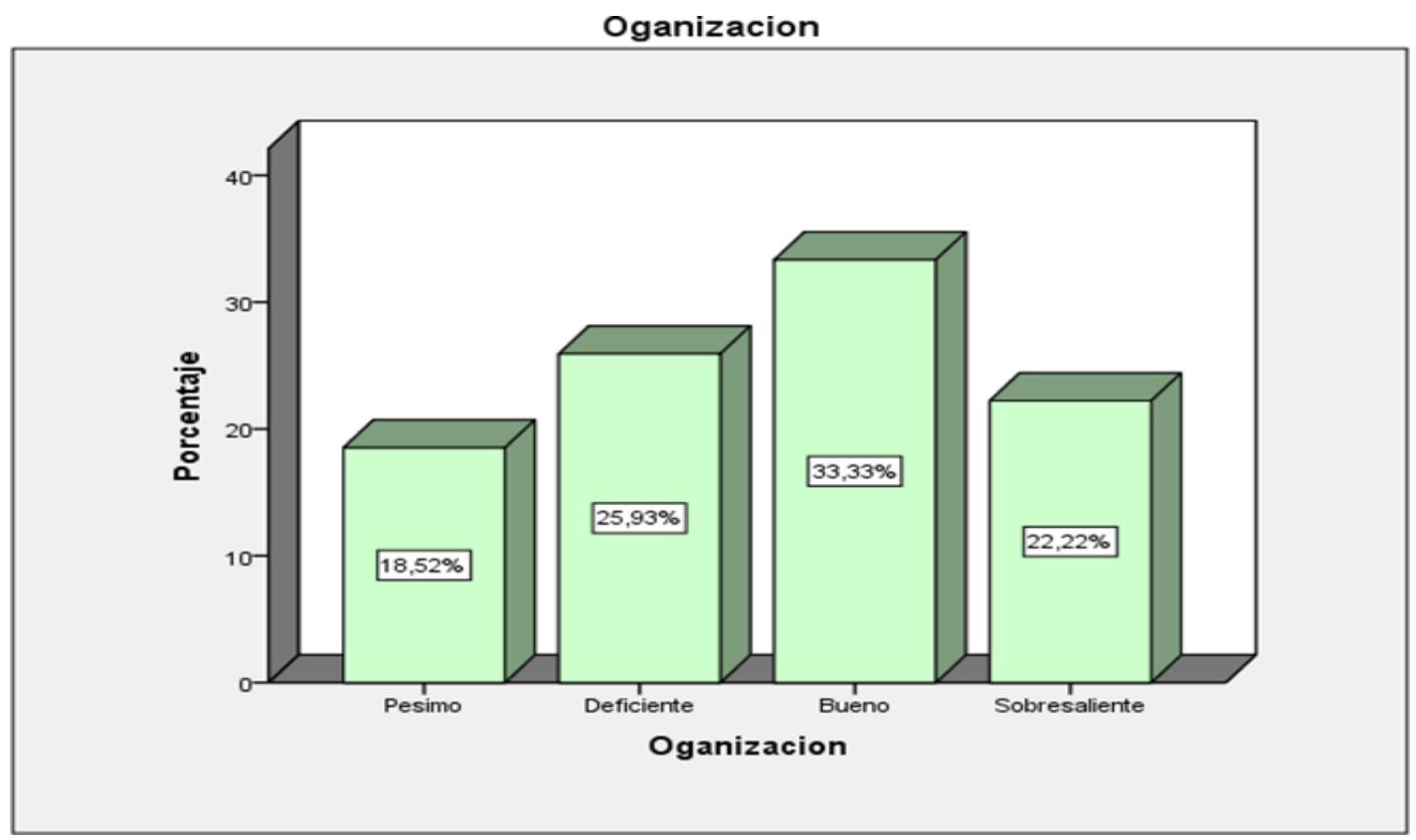

De acuerdo con la Figura 2 y la Tabla 10 de los datos, el 18,5\% lo considera pobre en términos de planificación, el 25,9\% deficiente, el 33,3\% bueno y el 22,2\% excepcional, y domina la relevancia para el aspecto organizativo. 


\section{Tabla 3}

Niveles de percepción de Dirección

\begin{tabular}{llcc}
\hline & Dirección & Frecuencia & Porcentaje \\
\hline \multirow{2}{*}{ Válido } & Pésimo & 25 & 23,1 \\
& Deficiente & 18 & 16,7 \\
& Sueno & 39 & 36,1 \\
& Sobresaliente & 26 & 24,1 \\
\hline
\end{tabular}

\section{Figura 3}

\section{Dirección}

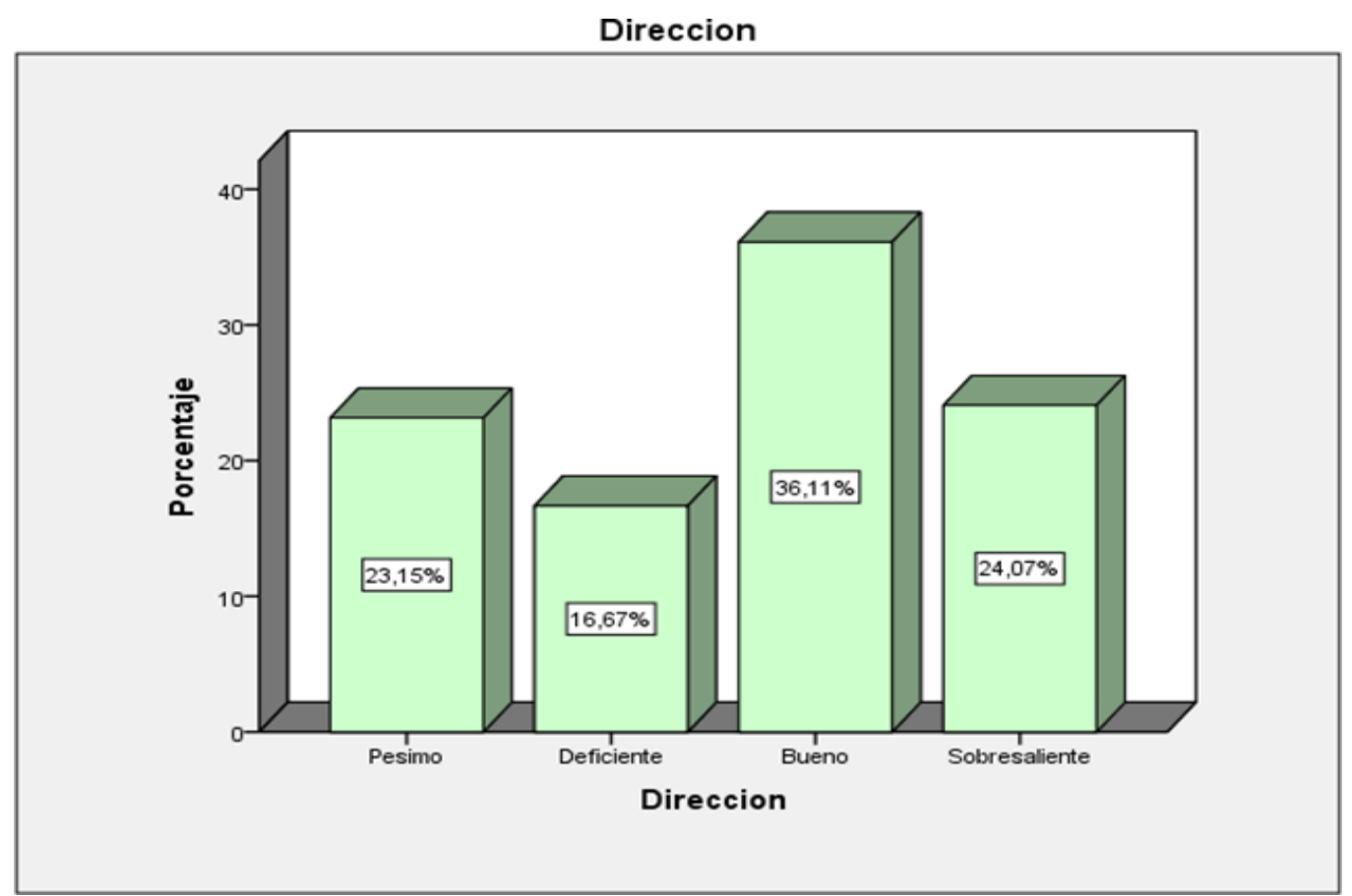

Según la Fig. 3 y la Tabla 11 de los datos, el 23,1\% la considera mala en términos de gestión, el $16,7 \%$ como mala, el $36,1 \%$ como buena y el $24,1 \%$ como especial, la importancia predomina en el lado de la dirección. 


\section{Tabla 4}

Niveles de percepción de Control

\begin{tabular}{clcc}
\hline \multicolumn{1}{c}{ Control } & Frecuencia & Porcentaje \\
\hline \multirow{4}{*}{ Válido } & Pésimo & 16 & 14,8 \\
& Deficiente & 27 & 25,0 \\
& Bueno & 40 & 37,0 \\
& Sobresaliente & 25 & 23,1 \\
& Total & $\mathbf{1 0 8}$ & $\mathbf{1 0 0 , 0}$ \\
\hline
\end{tabular}

\section{Figura 4}

\section{Control}

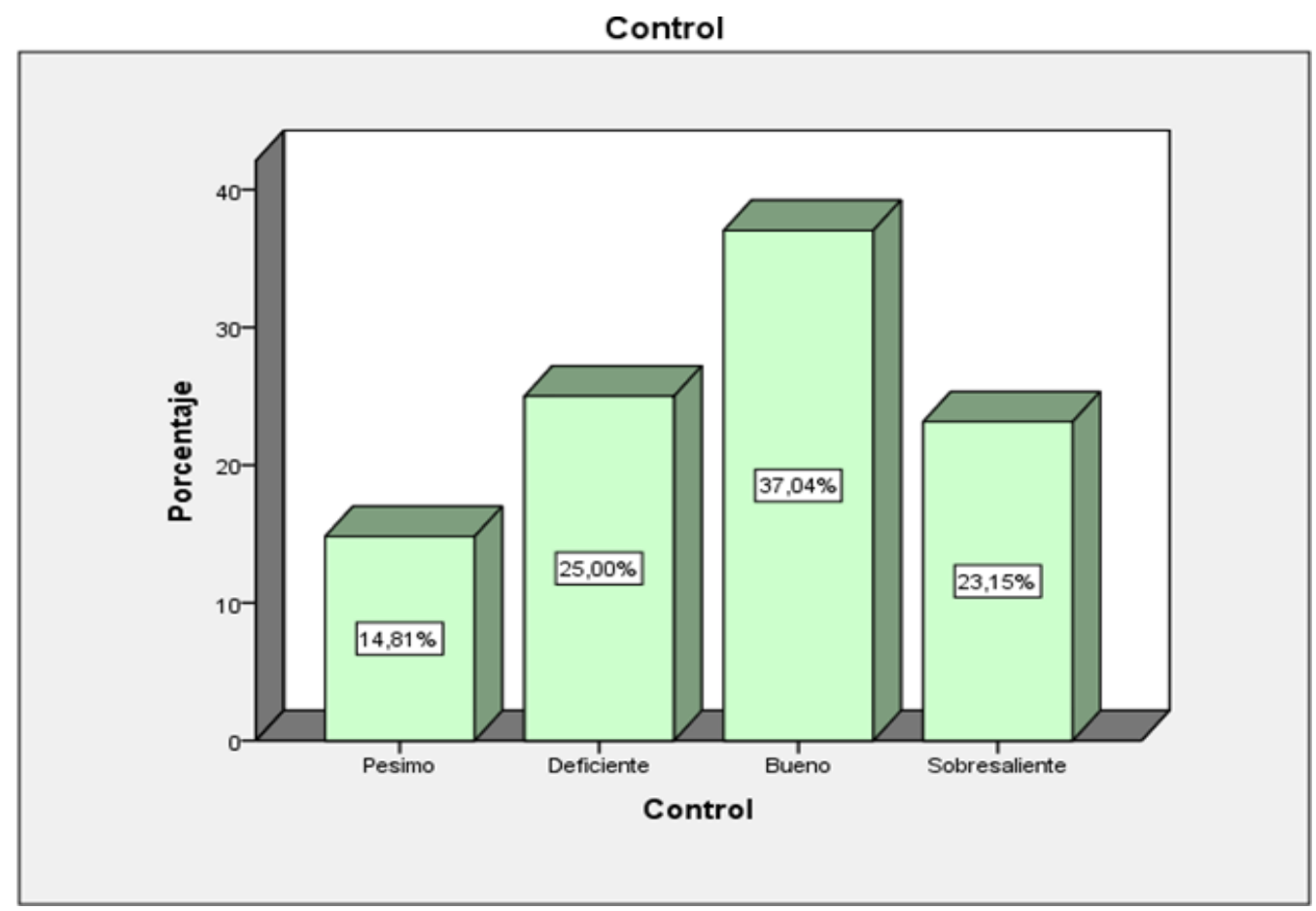

De acuerdo con la Figura 4 y Tabla 12 de los datos, el 14,8\% lo consideró pobre en la dimensión de control, el 25,0\% deficiente, el 37,0\% bueno y el $23,1 \%$ excepcional, y el grado de precisión predomina en la dimensión de control. 


\section{Tabla 5}

Niveles de percepción de Gestión municipal

\begin{tabular}{llcc}
\hline & Gestión municipal & Frecuencia & Porcentaje \\
\hline \multirow{2}{*}{ Válido } & Pésimo & 20 & 18,5 \\
& Deficiente & 20 & 18,5 \\
& Bueno & 42 & 38,9 \\
& Sobresaliente & 26 & 24,1 \\
& Total & $\mathbf{1 0 8}$ & $\mathbf{1 0 0 , 0}$ \\
\hline
\end{tabular}

\section{Figura 5}

Gestión municipal

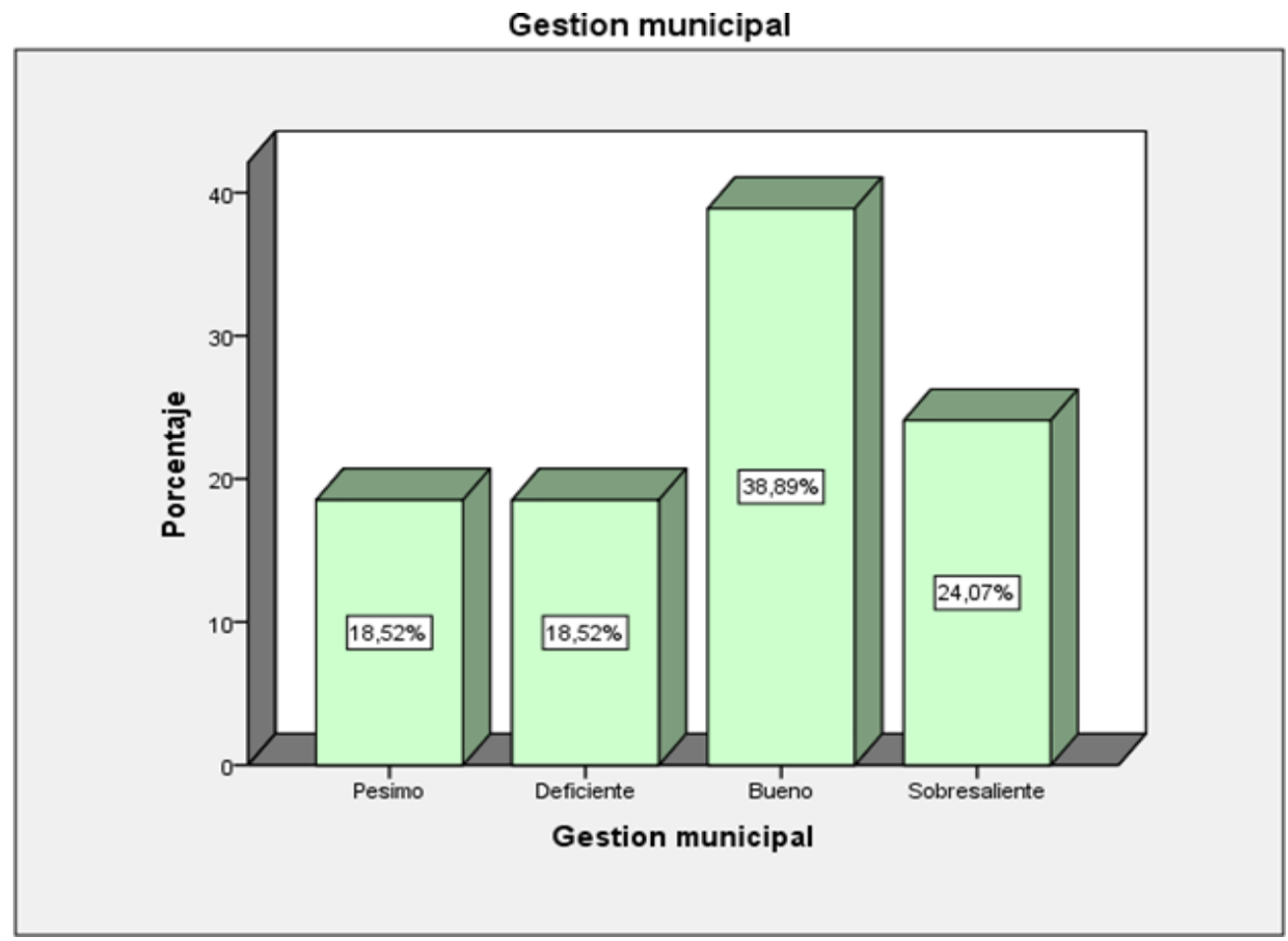

De acuerdo con la Figura 5 y la Tabla 13, el 18,5\% dijo que el nivel de gestión es malo a nivel de ciudad, el $18,5 \%$ no existe, el $38,9 \%$ es bueno, y el $24,1 \%$ en particular, el buen nivel de gestión prevalece a nivel municipal. 


\section{Tabla 6}

Niveles de percepción de Gobernabilidad

\begin{tabular}{clcc}
\hline & Gobernabilidad & Frecuencia & Porcentaje \\
\hline \multirow{2}{*}{ Válido } & Malo & 16 & 14,8 \\
& Regular & 22 & 20,4 \\
& Excelente & 45 & 41,7 \\
& Total & 25 & 23,1 \\
& & $\mathbf{1 0 8}$ & $\mathbf{1 0 0 , 0}$ \\
\hline
\end{tabular}

\section{Figura 6}

Gobernabilidad

\section{Gobernabilidad}

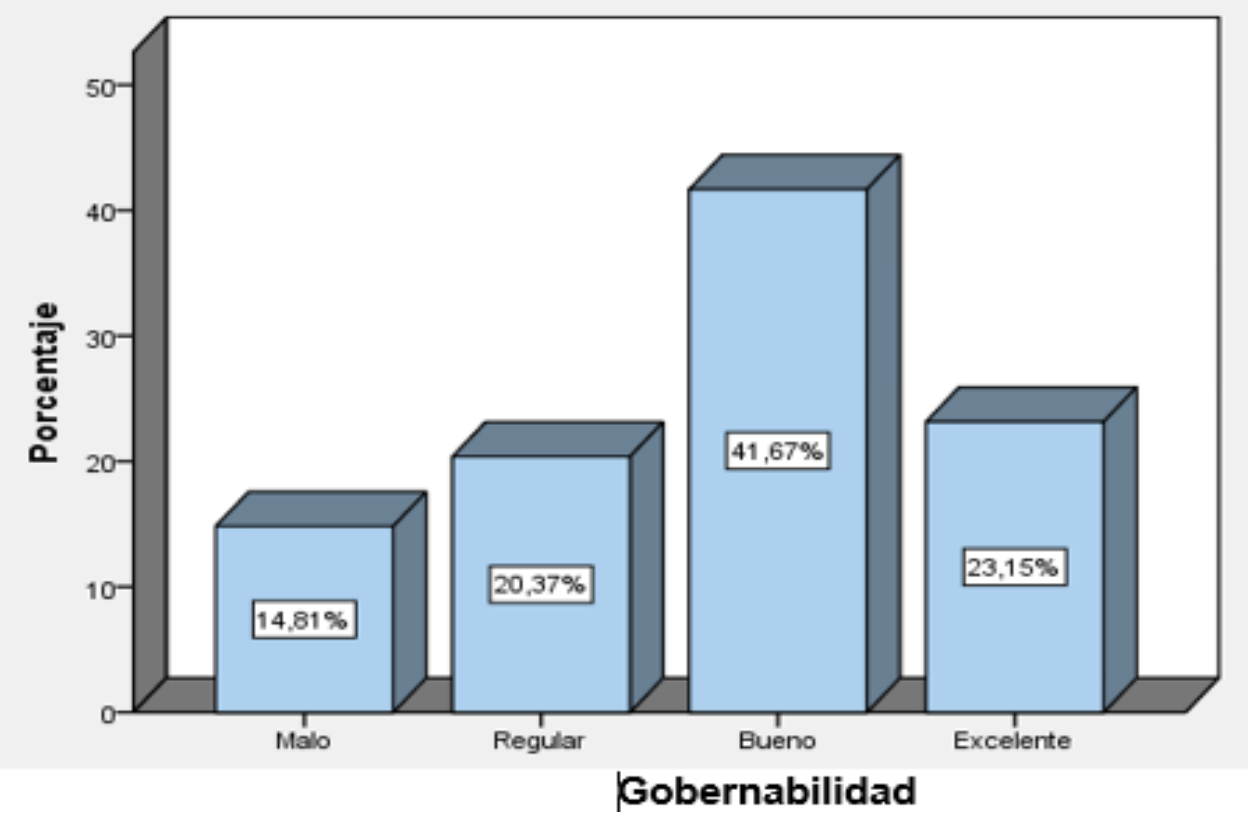

De acuerdo con la figura 6 y tabla 14 de datos, el $14.8 \%$ perciben un nivel malo en la dimensión Gobernabilidad, el 20.4\% un nivel regular, el $41.7 \%$ un nivel bueno y el $23.1 \%$ un nivel excelente siendo el nivel bueno el predominante en la dimensión Gobernabilidad. 


\section{Tabla 7}

Niveles de percepción de Voz y Rendición de Cuentas

\begin{tabular}{clcc}
\hline Cumplimiento & Frecuencia & Porcentaje \\
\hline \multirow{4}{*}{ Válido } & Malo & 10 & 9,3 \\
& Regular & 31 & 28,7 \\
& Bueno & 40 & 37,0 \\
& Excelente & 27 & 25,0 \\
& Total & 108 & 100,0 \\
\hline
\end{tabular}

\section{Figura 7}

Rendimiento de cuentas

\section{Voz y Rendición de Cuentas}

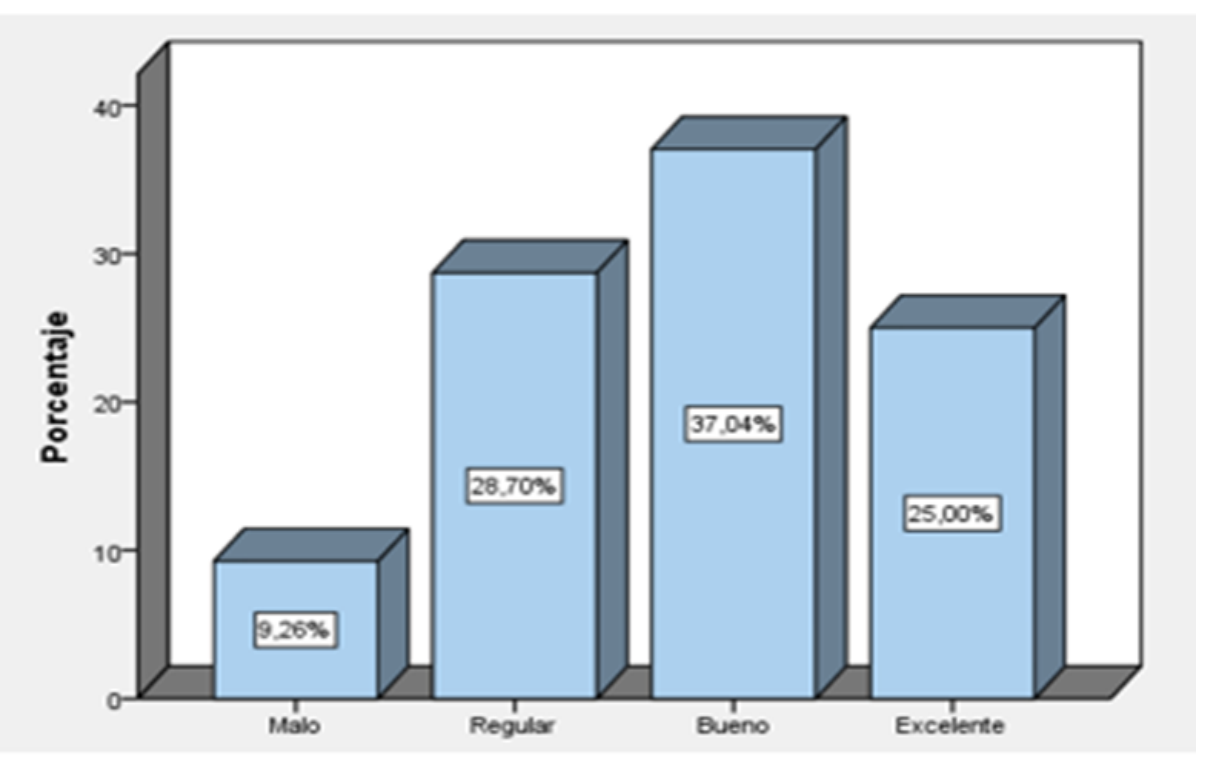

Voz y Rendición de Cuentas

De acuerdo con la figura 7 y tabla 15 de datos, el $9.3 \%$ perciben un nivel malo en la dimensión cumplimiento, el $28.7 \%$ un nivel regular, el $37.0 \%$ un nivel bueno y el $25.0 \%$ un nivel excelente siendo el nivel bueno el predominante en la dimensión cumplimiento. 


\section{Tabla 8}

Niveles de percepción de Estabilidad Política

\begin{tabular}{clcc}
\hline & Disciplinas & Frecuencia & Porcentaje \\
\hline \multirow{4}{*}{ Válido } & Malo & 25 & 23,1 \\
& Regular & 30 & 27,8 \\
& Bueno & 24 & 22,2 \\
& Excelente & 29 & 26,9 \\
& Total & $\mathbf{1 0 8}$ & $\mathbf{1 0 0 , 0}$ \\
\hline
\end{tabular}

\section{Figura 8}

Estabilidad política

\section{Estabilidad Política}

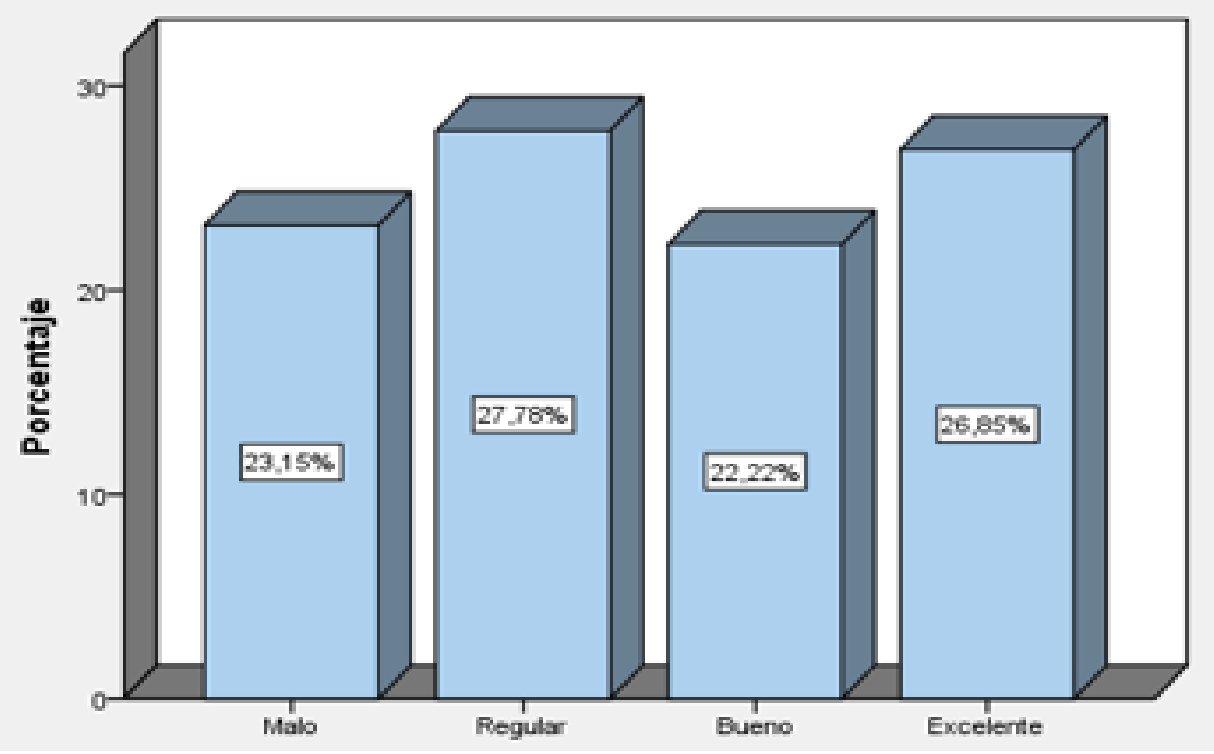

Estabilidad Política

De acuerdo con la figura 8 y tabla 16 de datos, el $23.1 \%$ perciben un nivel malo en la dimensión disciplinas, el $27.8 \%$ un nivel regular, el $22.2 \%$ un nivel bueno y el $26.9 \%$ un nivel excelente siendo el nivel excelente el predominante en la dimensión disciplinas. 


\section{Tabla 9}

Niveles de percepción de Imperio De La Ley

\begin{tabular}{cccc}
\hline \multicolumn{2}{c}{$\begin{array}{c}\text { Relaciones } \\
\text { interpersonales }\end{array}$} & Frecuencia & Porcentaje \\
\hline \multirow{4}{*}{ Válido } & Malo & 6 & 5,6 \\
& Regular & 24 & 22,2 \\
& Bueno & 41 & 38,0 \\
& Excelente & 37 & 34,3 \\
\hline
\end{tabular}

\section{Figura 9}

Imperio de la ley

\section{Imperio De La Ley|}

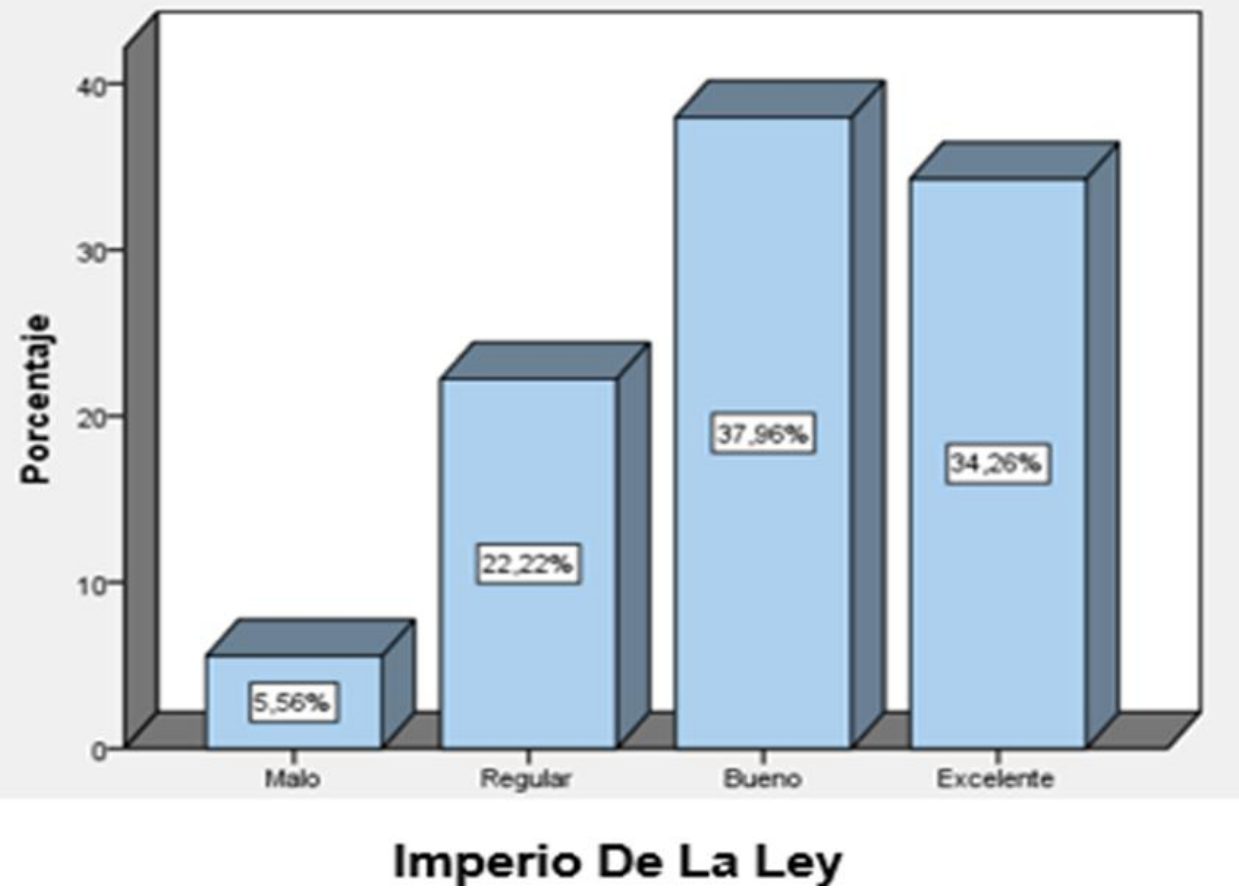

De acuerdo con la figura 9 y tabla 17 de datos, el 5.6\% perciben un nivel malo en la dimensión relaciones interpersonales, el $22.2 \%$ un nivel regular, el $38.0 \%$ un nivel bueno y el $34.3 \%$ un nivel excelente siendo el nivel bueno el predominante en la dimensión relaciones interpersonales. 
Ho: La Gestión Municipal no se relaciona significativamente con la gobernabilidad. La Gestión Municipal se relaciona significativamente con la gobernabilidad. El nivel de significación teórica 0.05 que corresponde a un nivel de confiabilidad de $95 \%$.

Tabla 10

Coeficiente de correlación de Spearman: Gobernabilidad y atención comunitaria

\begin{tabular}{|c|c|c|c|c|}
\hline \multicolumn{2}{|c|}{ Correlaciones } & & & $\begin{array}{c}\text { Atención } \\
\text { comunitaria }\end{array}$ \\
\hline \multirow{6}{*}{$\begin{array}{l}\text { Rho de } \\
\text { Spearman }\end{array}$} & \multirow{6}{*}{ Gobernabilidad } & $\begin{array}{l}\text { Coeficiente de } \\
\text { correlación }\end{array}$ & \multirow[t]{2}{*}{1,000} &, $823 * *$ \\
\hline & & Sig. (bilateral) & & ,000 \\
\hline & & $\mathrm{N}$ & 108 & 108 \\
\hline & & $\begin{array}{l}\text { Coeficiente de } \\
\text { correlación }\end{array}$ &, $823 * *$ & 1,000 \\
\hline & & Sig. (bilateral) & ,000 & $\cdot$ \\
\hline & & $\mathrm{N}$ & 108 & 108 \\
\hline
\end{tabular}

**. La correlación es significativa en el nivel 0,01 (bilateral).

Decisión estadística: El coeficiente de correlación Rho de Spearman evidencia una relación estadísticamente significativa, muy alta $(\mathrm{r}=0.823)$ y directamente proporcional, entre Gobernabilidad y atención comunitaria; asimismo, debido a que el p_valor $<0.05$ se rechaza la hipótesis nula, y se acepta la hipótesis general.

Ho: La planificación de la Gobernabilidad no se relaciona significativamente con la atención comunitaria

H1: La planificación de la Gobernabilidad se relaciona significativamente con la atención comunitaria.

\section{Tabla 11}

Gobernabilidad y Planificación

\begin{tabular}{|c|c|c|c|c|}
\hline \multicolumn{2}{|r|}{ Correlaciones } & & Planificación & Gobernabilidad \\
\hline \multirow{7}{*}{$\begin{array}{l}\text { Rho de } \\
\text { Spearman }\end{array}$} & Planificación & $\begin{array}{l}\text { Coeficiente } \quad \text { de } \\
\text { correlación }\end{array}$ & 1,000 & $829 * *$ \\
\hline & \multirow{6}{*}{ Gobernabilidad } & Sig. (bilateral) & . & ,000 \\
\hline & & $\mathrm{N}$ & 108 & 108 \\
\hline & & Coeficiente &, $829 * *$ & 1,000 \\
\hline & & correlación & & \\
\hline & & Sig. (bilateral) &, 000 & . \\
\hline & & $\mathrm{N}$ & 108 & 108 \\
\hline
\end{tabular}

**. La correlación es significativa en el nivel 0,01 (bilateral). 
El coeficiente de correlación Rho de Spearman evidencia una relación estadísticamente significativa, muy alta $(\mathrm{r}=0.829)$ y directamente proporcional, entre planificación y la gobernabilidad; asimismo, debido a que el p_valor $<0.05$ se rechaza la hipótesis nula, y se acepta la hipótesis específica.

Ho: La organización de la gestión municipal no se relaciona significativamente con la gobernabilidad.

H1: La organización de la gestión municipal se relaciona significativamente con la gobernabilidad.

\section{Tabla 12}

Coeficiente de correlación de Spearman: Organización y la Gobernabilidad

\begin{tabular}{|c|c|c|c|c|}
\hline & Correlaciones & & Organización & Gobernabilidad \\
\hline \multirow{7}{*}{$\begin{array}{l}\text { Rho de } \\
\text { Spearman }\end{array}$} & \multirow{3}{*}{ Organización } & $\begin{array}{l}\text { Coeficiente de } \\
\text { correlación }\end{array}$ & 1,000 &, $802 * *$ \\
\hline & & Sig. (bilateral) & . &, 000 \\
\hline & & $\mathrm{N}$ & 108 & 108 \\
\hline & \multirow{4}{*}{ Gobernabilidad } & Coeficiente de & & \\
\hline & & correlación &, $802 * *$ & 1,000 \\
\hline & & Sig. (bilateral) & ,000 & . \\
\hline & & $\mathrm{N}$ & 108 & 108 \\
\hline
\end{tabular}

**. La correlación es significativa en el nivel 0,01 (bilateral).

El coeficiente de correlación Rho de Spearman muestra una correlación altamente significativa $(r=0,802)$ y una relación positiva entre organización y juicio; De manera similar, dado que p_value $<0.05$, se rechaza la hipótesis nula y se acepta la hipótesis dada. Ho: La dirección de la gestión municipal no se relaciona significativamente con la gobernabilidad.

H1: La dirección de la gestión municipal se relaciona significativamente con la Gobernabilidad

\section{Tabla 13}


Coeficiente de correlación de Spearman: Dirección y la Gobernabilidad.

\begin{tabular}{|c|c|c|c|c|}
\hline & Correlaciones & & Dirección & Gobernabilidad \\
\hline \multirow{6}{*}{$\begin{array}{l}\text { Rho de } \\
\text { Spearman }\end{array}$} & \multirow{3}{*}{ Dirección } & $\begin{array}{l}\text { Coeficiente de } \\
\text { correlación }\end{array}$ & 1,000 &, $821 * *$ \\
\hline & & Sig. (bilateral) & $\cdot$ & ,000 \\
\hline & & $\mathrm{N}$ & 108 & 108 \\
\hline & \multirow{3}{*}{ Gobernabilidad } & $\begin{array}{l}\text { Coeficiente de } \\
\text { correlación }\end{array}$ &, $821 * *$ & 1,000 \\
\hline & & Sig. (bilateral) & ,000 & . \\
\hline & & $\mathrm{N}$ & 108 & 108 \\
\hline
\end{tabular}

**. La correlación es significativa en el nivel 0,01 (bilateral).

El coeficiente de correlación Rho de Spearman muestra una relación estadísticamente significativa muy alta $(\mathrm{r}=0,821)$ y una relación positiva entre gestión y desempeño laboral; De manera similar, dado que p_value $<0.05$, se rechaza la hipótesis nula y se acepta la hipótesis dada.

Ho: El control de la gestión municipal no se relaciona significativamente con la Gobernabilidad.

H1: El control de la gestión municipal se relaciona significativamente con la Gobernabilidad.

\section{Tabla 13}

Coeficiente de correlación de Spearman: Control y la Gobernabilidad.

\begin{tabular}{lllcc}
\hline & Correlaciones & & Control & Gobernabilidad \\
\hline & & Coeficiente de & 1,000 &, $786^{* *}$ \\
Control & correlación & &, 000 \\
Rho de & & Sig. (bilateral) & 108 & 108 \\
Spearman & & $\mathrm{N}$ & 108 & \\
& & Coeficiente de & & 1,000 \\
& \multirow{2}{*}{ Gobernabilidad } & correlación &, $786^{* *}$ &. \\
& & Sig. (bilateral) &, 000 & 108 \\
\hline
\end{tabular}

**. La correlación es significativa en el nivel 0,01 (bilateral).

El coeficiente de correlación Rho de Spearman evidencia una relación estadísticamente significativa, alta $(\mathrm{r}=0.786)$ y directamente proporcional, entre control y gobernabilidad; 
asimismo, debido a que el p_valor $<0.05$ se rechaza la hipótesis nula, y se acepta la hipótesis específica.

\section{DISCUSIÓN}

Se destacaron los resultados obtenidos en el proceso de aplicación de la tecnología de estadística predictiva con SPSS V23 para obtener estadística descriptiva de las variables de investigación y delimitar las hipótesis según el problema y el objetivo de la investigación.

El objetivo general propuesto es determinar la relación que existe entre la administración de la ciudad que no está significativamente relacionada con la gobernanza, para lograr los objetivos de la investigación, se aplicaron las herramientas de las variables. La planificación de la gestión de la ciudad está estrechamente relacionada con la gobernanza. Como plantea Jaramillo (2022), Se han puesto en marcha reformas institucionales debido a la incorporación de Colombia a la Organización para la Cooperación y el Desarrollo Económico (OCDE). Una de ellas está asociada al componente de "gobernanza normativa o regulatoria" destinado a mejorar el proceso de toma de decisiones. Montalvo (2016), la municipalidad puede contribuir en el desarrollo de los sectores poblaciones menos favorecidos, si se convierte en un ente coordinador entre los actores locales, capaz de identificar oportunidades existentes en el mercado interno generar sinergias con potenciales proveedores, provenientes especialmente del del Distrito. Espinoza (2017) Utilizo Likert en su encuesta realizado a 103 pobladores para su muestra quienes fueron elegidos al azar para dicho estudio, llego a la conclusión: Existe una relación directa entre la gobernabilidad y el desarrollo local en la Municipalidad provincial de Ica, 2016. Oxxo (2014), teniendo como objetivo el decretar una concordancia entre las variables y la relación con los procesos de planificación, integración y desarrollo.

Siendo así que para mejorar la efectividad de la organización se utilicen estratégicamente todas estas actividades. (Slocum et al., 2009).

Estas herramientas se desarrollaron a partir de las dimensiones e indicadores de las variables de investigación. Contrariamente a la suposición general, en la Tabla 19, el coeficiente de correlación Rho de Spearman muestra una relación estadísticamente significativa muy alta $(\mathrm{r}=0,829)$ y una relación positiva entre planificar y realizar el trabajo; De manera similar, dado que p_value $<0.05$, se rechaza la hipótesis nula y se acepta la hipótesis dada. En el contraste de la hipótesis específica 1, en la tabla 20, el 
coeficiente de correlación Rho de Spearman evidencia una relación estadísticamente significativa, alta $(\mathrm{r}=0.802)$ y directamente proporcional, entre organización y la gobernabilidad; asimismo, debido a que el palor $<0.05$ se rechaza la hipótesis nula, En contraste con la hipótesis 2 seleccionada, en la Tabla 21, el coeficiente de correlación Rho de Spearman muestra una relación estadísticamente significativa muy alta $(r=0.821)$ y una relación positiva entre administración y manejo, debido a que p_value $<0.05$, la hipótesis nula es rechazada y la especificada Se acepta la hipótesis. 2. Tejeda, LC (2014) siguió de cerca la tesis titulada "Gobernanza y Mejoramiento Administrativo en el Distrito Urbano del Distrito Bellavista Callao", concluyendo que: La modernización administrativa como proceso de innovación solo puede reconocerse en el nivel de confianza de los funcionarios y personal, importante para iniciar el desarrollo de la capacidad de gestión. (p. 127) "Concluyó que existe un alto grado de relación entre la gestión de habilidades y la gestión, y los empleados de la ciudad deben estar capacitados para hacer uso de sus habilidades. En contraste con la hipótesis específica 3, en la Tabla 22, el coeficiente de correlación Rho de Spearman muestra una relación altamente positiva y estadísticamente significativa $(r=0,786)$ entre control y juicio; De manera similar, dado que p_value $<0.05$, se rechaza la hipótesis nula y se acepta la hipótesis dada. 3; De igual forma, Chiavenato (2002) coincide en que el control actualmente consta de dos procesos, es decir, acompañado de un seguimiento y dirección de la plena implementación del logro de la gobernanza basada en objetivos. Por tanto, como objetivos específicos, partiendo de la administración pública, se determina que el control es verificar y motivar a la administración del gobierno para ver si las tareas asignadas están cumplidas y son adecuadas. A partir de este enfoque, se determina que el control incluye monitorear y evaluar el desempeño de la organización y tomar las medidas necesarias para lograr la eficiencia en el desempeño de las funciones.

\section{CONCLUSIÓN O CONSIDERACIONES FINALES}

Al hacer el contraste de la hipótesis general usando técnica estadística predictiva del SPSS V23, existen evidencias suficientes para afirmar que la gobernabilidad tiene relación positiva y significativa con la gestión municipal $(\mathrm{rs}=0.823, \mathrm{p}<0.05)$. Una Administración fuerte no debería ser una Administración sobredimensionada, intrusiva o gigantesca, sino firme en la defensa del bien común, independiente frente a grupos de interés espurios y dinámica para impulsar el desarrollo de sus territorios. Todo ello es 
posible y necesario para afrontar los retos del nuevo siglo; y todo ello es compatible con dejar atrás modelos obsoletos que ya no tienen sentido.

Al hacer el contraste de la hipótesis específica 1 usando técnica estadística predictiva del SPSS V23, existen evidencias suficientes para afirmar la planificación tiene relación positiva y significativa con la gobernabilidad ( $\mathrm{rs}=0,829, \mathrm{p}<0.05)$. La Administración no puede prestar los servicios de la misma manera que antes porque la ciudadanía exige otro tipo de relación. Hoy se pide mayor agilidad en los trámites y más flexibilidad orgánica y funcional en todos los ámbitos en los que lo público interactúa con la ciudadanía.

En el contraste de la hipótesis específica 2 usando técnica estadística predictiva del SPSS V23, existen evidencias suficientes para afirmar que la organización tiene relación positiva y significativa con la gobernabilidad ( $r s=0.802, \mathrm{p}<0.05)$. Con el fin de motivar a los profesionales de la Administración Pública en el desarrollo de su labor, conviene establecer su carrera administrativa, de manera que puedan acceder a escalas superiores mediante determinados mecanismos de promoción interna. Para ello es necesario desarrollar y aplicar el principio de evaluación

Al hacer el contraste de la hipótesis específica 3 usando técnica estadística predictiva del SPSS V23, existen evidencias suficientes para afirmar que la dirección tiene relación positiva y significativa con la gobernabilidad ( $r s=0.821, \mathrm{p}<0.05)$. De nuevo y al margen del debate sobre el tamaño, es preciso tener en cuenta que necesitamos un sector público con un papel bien definido y que funcione adecuadamente

Al hacer el contraste de la hipótesis específica 4 usando técnica estadística predictiva del SPSS V23, existen evidencias suficientes para afirmar que el control tiene relación positiva y significativa con la gobernabilidad $(r s=0.786, p<0.05)$. Del sector público depende, directa o indirectamente, la educación, la sanidad, la seguridad, las infraestructuras, el trabajo, la competitividad o el entorno en el que vivimos y trabajamos. Del sector público depende en gran medida la calidad del aire que respiramos o la salubridad del agua que bebemos. Y del sector público depende que las sociedades puedan progresar salvaguardando la justicia, la seguridad y la libertad de los individuos. Todavía hay quien se pregunta por qué hay que transformar la Administración Pública.

\section{LISTA DE REFERENCIAS}


Alcántara, m. (2014). Gobernabilidad, crisis y cambio, Centro de Estudios Constitucionales,Madrid, http://www.iapqroo.org.mx/.pdf

Ander-EGG, Ezequiel. “Técnicas de investigación social” Ed. Humanistas. Buenos Aires.

Arias, F. (2006). Proyecto de investigación: introducción a la metodología científica (5 ed.) Caracas: Espíteme.

Barroso Orellana, Ana Paulina (2016). La gobernabilidad vista desde el liderazgo socio organizativo de la asociación de mujeres Waorani de la amazonia ecuatoriana Amwae (2010-2012). http://repositorio.puce.edu.ec/handle/22000/11064

Bernal, E. (2006) Metodología de la investigación. Colombia: Pearson Educación

Camou, Antonio (Estudio preliminar y compilación) (2001). Los desafíos de la

Chiavenato, I. (2002). Gestión del Talento Humano. Bogotá: McGraw Hill.

Espinoza Delgado, H. A. (2017). Gobernabilidad y desarrollo local en la Municipalidad Provincial de Ica, 2016. Universidad César Vallejo.

Gobernabilidad. México: Flacso/IISUNAM/Plaza y Valdés.

Gutiérrez Simón, R. (2017). Jorge Giraldo Ramírez, "Las ideas en la guerra. Justificación y crítica en la Colombia contemporánea\&quot, Bogotá, Debate, 2015, 240 pp. Res Publica. Revista de Historia de las Ideas Políticas, 20(1), 187189. https://doi.org/10.5209/RPUB.54902

Hernández Sampieri, R, Fernández, C \& Baptista, P. (2010). Metodología de la Investigación. (Quinta Edición). México D.F, México: McGraw-Hill.

Hernández, Fernández y Baptista (2014), Metodología de la investigación. México: Mc Graw Hill.

http://repositorio.puce.edu.ec/handle/22000/10563

http://www.institut-gouvernance.org/es/analyse/fiche-analyse-334.html

Jaramillo, L. G., \& Jaramillo, J. I. P. (2022). Citizen participation in local regulatory governance processes. analysis of normative production in the municipality of medellín (colombia) (2017-2018). [Participación ciudadana en procesos de gobernanza regulatoria local. Análisis de la construcción normativa en la Alcaldía de Medellín (Colombia) (2017-2018)] Revista Derecho Del Estado, (51), 103135. doi:10.18601/01229893.n51.04

Mayorga, F. \& Córdova, E., 2007, “Gobernabilidad y Gobernanza en América latina”, Working Paper NCCR Norte-Sur IP8, Ginebra. No publicado. 
Menendez, A: ' Validez, confiabilidad y utilidad. Disponible en http://www.gobierno.pr/nr/rdonlyres/ $5 c f 112 b b-5811-4 a 9 a-8 d 1 e-$ 1ba213c5eef7/0/14validez.pdf, octubre 2016.

Montalvo Carrión, María Lorena (2016). La economía popular en el Distrito metropolitano de Quito: una propuesta alternativa para su desarrollo productivo

Prats, J. "Hacia una definición de la Investigación en Didáctica de las Ciencias Sociales". En: [I] congreso nacional de didácticas específicas. las didácticas de las áreas curriculares en el siglo XXI. Granada: Grupo Editorial Universitario. 2001.

Rodríguez, C. y Gutiérrez, J. (2005). "Un modelo de validación de estudios empíricos en investigación educativa mediante procedimientos de triangulación. Aplicación a un estudio de caso sobre disfunciones y desajustes asociados a la reforma de un plan de estudios universitario", Revista Portuguesa de Pedagogía, (en prensa).

Ruiz Bolívar, Carlos (2002). Instrumentos de investigación educativa. Procedimientos para su diseño y validación. Barquisimeto, Venezuela: CIDEG. 266 p.

Sierra, R. (1994). Técnicas de investigación social. Madrid: Paraninfo.

Slocum, J. W., \& Hellriegel, D. (2009). Principles of organizational behavior. Mason, $\mathrm{OH}$ : South-Western Cengage Learning.

Stoner, G., Freeman, N y Gilbert, T. (2009). Administración de empresas. Barcelona: Herder.

Tamayo, M. (2003). El proceso de la investigación científica. México, D.F., Limusa. Noriega Editores.

Tejeda, L. C. (2014). Gestión administrativa y su mejora en la municipalidad distrital Bellavista. Callao. (Tesis de maestría, Universidad Nacional del Callao). Lima, Perú.

Vargas, Z. (2009). La investigación aplicada: una forma de conocer las realidades con evidencia científica. Revista Educación, 33(1), 155-165.

Yadira Corral (2008). Diseño de cuestionarios para recolección de datos. Revista ciencias de la educación. Año 2010 / Vol. 20 / Nº 36 / Valencia, Julio - Diciembre. 